\title{
Filosofía franciscana en Chile: dos principales exponentes
}

\author{
Jaime Caiceo Escudero' \\ Universidad de Santiago de Chile (Chile)
}

Recibido: agosto 8 de 2019. Revisado: septiembre: 16 de 2019. Aceptado: noviembre 15 de 2019

Referencia norma APA: Caicedo, J. (2019). Filosofía franciscana en Chile: dos principales exponentes. Rev. Guillermo de Ockham, 17(2), 81-90.

doi: https://doi.org/10.21500/22563202.4365

\section{Resumen}

La orden franciscana está presente en Chile desde 1553 y en este artículo se destacan dos principales filósofos: Alfonso Briceńo Arias (1587-1668) y Eduardo Rosales Rojas (1906-1970). Al primero se lo conoce como el segundo Escoto, pues su pensamiento se inspira en el famoso beato inglés. El segundo se doctora en la Universidad Católica de Chile, en donde será académico de Teología moral.

El objeto de este escrito es desarrollar el pensamiento filosófico de ambos pensadores franciscanos chilenos. Este trabajo es fruto de una investigación más amplia acerca de la filosofía franciscana. Para realizarla se ha recurrido a la —metodología histórica — revisando fuentes primarias y secundarias—y al análisis filosófico de los escritos de los pensadores.

Palabras clave: filosofía franciscana, filosofía aristotélica, filosofía agustiniana, escolástica, pensamiento chileno.

\section{Franciscan Philosophy in Chile: Two Main Exponents}

\section{Abstract}

The Franciscan Order has been present in Chile since 1553 and this article includes two main philosophers: Alfonso Briceño Arias (1587-1668) and Eduardo Rosales Rojas (1906-1970). The first is known as the Second Escoto since his thinking is inspired by the famous English theologists (John Duns Scotus). The second one, Phd from the the Catholic University of Chile where he would eventually become an academic of Moral Theology.

The purpose of this article is to analyze the philosophical thinking of both Chilean Franciscan theorists. This work is the result of a broader research on Franciscan Philosophy. To carry it out, the historical methodology was used reviewing primary and secondary sources as well as the philosophical analysis of the thinkers' writings.

Keywords: Franciscan Philosophy, Aristotelian Philosophy, Augustinian Philosophy, Scholastic, Chilean Thought.

1. Académico de Post-Grado del Departamento de Educación. Correo electrónico: jcaiceo@hotmail.com 


\section{Introducción}

San Francisco nació en Asís a finales del siglo XII y fundó la orden en su ciudad natal en $1208 .^{2}$ Su característica distintiva fue proseguir las enseñanzas del Evangelio de Jesucristo (Caiceo, 2018); es decir, llevar una vida austera, centrada en los más necesitados y con una misión clara de predicar las enseñanzas del Divino Maestro (Directorio Franciscano, s/f). Por su sello distintivo se le conoce como orden mendicante.

Respecto al espíritu de san Francisco, un fraile chileno señala:

Como se dejó conducir por los deseos de su corazón, y teniendo en cuenta una certeza que va a acompañar más tarde a los pensadores franciscanos, en lo profundo del corazón del hombre habita Dios, Francisco al igual que nosotros en un momento que nadie más que Dios conoce se encontró con el huésped de su corazón, que lo hizo dar un giro copernicano, abandonando los sueños de caballero para abrazar el sueño utópico de hacer vida el Evangelio, desde este momento -difícil para mí de precisar cronológicamente en la vida de Francisco-, comenzó a vivir la forma y vida del Santo Evangelio (cf. Regla Bulada de San Francisco I,

1) (Escárate, 2014 p. 142).

El filósofo fray Alfonso Briceño, aunque desarrolló su labor fundamentalmente en el extranjero y muere como obispo en Caracas, nació en Chile y aquí vivió sus primeros años. Su principal obra es Celebriores controversias in primum Sententiarum Ioannis Duns Scoti, Doctoris subtilis Theologorum facile Principis..., ${ }^{3}$ la cual se comentará más adelante.

fray Eduardo Rosales, por su parte, nace y muere en Chile y su tesis doctoral se titula Doctrina acerca de la limosna según san Buenaventura y su principal obra es Hacia una moral social y profesional en dos tomos, publicada por la Editorial Universitaria. El contenido de esta última se explicará posteriormente.

Tal como se indicó en el resumen, este artículo se basa en una investigación más amplia sobre la Filosofía franciscana y ya se ha publicado un libro en dos ediciones (Caiceo, 2014 y 2018) y un artículo (Caiceo, 2016).

\section{El origen de la filosofía franciscana en Europa y América}

La Filosofía franciscana surgió en Bolonia, Italia, a comienzos del siglo XIII por razones diversas: fundamentar el pensamiento cristiano frente a los errores de la época y formar a sus religiosos. Por lo mismo, se extendieron sus centros universitarios a las ciudades relevantes de la época: Oxford y Cambridge en Inglaterra y París en Francia (Caiceo, 2016).

Así como surgió la filosofía en Europa, algo similar ocurrirá a partir del descubrimiento de América, puesto que los franciscanos vendrán con los conquistadores y se destacarán por su defensa de los indígenas. Entre ellos,cabe mencionar a san Francisco Solano, el beato Sebastián de Aparicio y fray Luis de Bolańos. Entre los principales filósofos chilenos se destacan Alfonso Briceño Arias (1587-1668) y Eduardo Rosales Rojas (1906-1970), que se expondrán en los puntos siguientes.

\section{Alfonso Briceño. Vida y pensamiento}

\section{Algunos rasgos biográficos}

Alonso, Alfonso o Ildefonso Briceño Arias, nace en Santiago de Chile en 1587, hijo primogénito de padres españoles: el capitán Alonso Briceño de Arévalo, oriundo de Guadalajara, España, y Jerónima Arias de Córdoba, natural de Santiago. Según Urdaneta (1973, p. 57) "hijo, nieto y bisnieto de conquistadores del Perú". A los cincoaños de edad sus padres lo llevan a Lima para que estudie con los franciscanos del convento San Francisco, el Grande. Ante la muerte de sus padres y por el ejemplo y motivación de sus formadores, ingresa el 30 de enero de 1605 a la orden franciscana, profesa en febrero de 1606 (Manzano, 1992). Un cronista contemporáneo suyo describe estos hechos, así:

El padre fray Alonso de Briceño fue profesor de filosofía en Lima en virtud de un certamen que ganó. El 30 de enero de 1605 tomó el hábito en la ciudad de Lima y fue contado entre los seguidores de san Francisco. Después de trece meses profesó en manos de fray Benito Huertas (citado por Manzano, 1992, p. 336).

2. Existen diferentes versiones sobre el año del surgimiento de la orden: 1208 corresponde al ańo en que en la capilla de Santa María de los Ángeles recibió un llamado divino: "No lleven monedero, ni bolsón, ni sandalias...”. 1209 corresponde al año en que san Francisco va a Roma con sus primeros seguidores a solicitarle al papa Inocencio III la aprobación de la Regla de la Orden. 1215 en el Concilio de Letrán se consolida la orden (Caiceo, 2018).

3. Las célebres controversias en las primeras sentencias de Juan Duns Escoto, Doctor Sutil, Príncipe de los Teólogos

$82<$ Universidad de San Buenaventura, Cali, Colombia 
A pesar de haber vivido solamente cinco años en Chile y haber venido posteriormente en calidad de comisario y visitador de la orden por corto tiempo, para fray Alonso Briceño fue importante su lugar de nacimiento y el recuerdo plácido de su nińez entre el cerro Santa Lucía y las riberas del río Mapocho, pues siempre después de su nombre indicaba su condición de chileno.

Inicia su carrera como profesor en Lima en 1613, luego de una profunda formación filosófica y teológica que lo transforma en un gran conocedor de la filosofía antigua y medieval, de la Patrística, el misticismo y la escolástica. Existen diferentes versiones respecto a si él hace sus estudios de filosofía antes de entrar al convento o dentro de él (Muñoz, 2004). Según un testigo de la época “(...) desde su juventud sobresalió entre todos sus compañeros por la sutileza y penetración de su ingenio" (Sotomayor, en Manzano, 1992 p. 339). Participa en concurso de oposición para la cátedra de filosofía, con presencia de "(...) lúcidos sujetos y ganó la oposición, obteniendo el primer puesto en el certamen" (Urdaneta, 1973: p. 60). Durante quince ańos ejerce la docencia. Durante los tres primeros dicta yilosofía y los doce restantes enseńa teología. Por su gran capacidad intelectual en el ejercicio de la docencia es nombrado profesor lector, bis jubilatus" . ${ }^{4}$ Él mismo lo señala en la primera parte de su principal obra acerca de los comentarios a las Sentencias de Duns Escoto: "Authore Patre F. Ildephonso Brizeño, Chilensi Sacrae Theologiae Professore bis jubilato..." (Manzano, 1992, p. 344). Este rango lo han tenido grandes personalidades intelectuales de la orden franciscana como san Buenaventura y el beato Juan Duns Escoto. En la América de la época solo lo obtiene el chileno fray Alonso Briceño. Por su gran erudición y conocimiento de la filosofía escotista, es conocido como “el segundo Escoto" (Urdaneta, 1973, p. 61).

Durante su magisterio en Lima es designado guardián ${ }^{6}$ del Colegio Franciscano y consejero de la provincia en Lima. Una vez retirado de la docencia, es nombrado comisario y visitador ${ }^{7}$ de la provincia de la Santísima Trinidad en Chile. En su visita, probablemente en 1628, celebra un capítulo provincial y preside la elección de los Superiores. Luego, a lomo de mula atraviesa la cordillera de los Andes para visitar la provincia de San Antonio de los Charcas en Bolivia. El convento principal se ubicaba en La Plata, hoy Sucre y desde allí insta en 1629 a sus hermanos a la predicación a los indios habitantes en las quebradas y lugares más apartados (Urdaneta, 1973). Prosigue su periplo por las montańas y llega al valle de Jauja, en zona peruana, lugar que Francisco Pizarro quiso erigir como capital del futuro Virreinato del Perú. En este lugar ejerce el cargo de vicario provincial. ${ }^{8}$ Posteriormente se traslada a Cajamarca, ochocientos kilómetros al norte de Lima, en donde ejerce como guardián del convento del lugar y prosigue como vicario provincial. Las crónicas de la época relatan que "las excelentes disposiciones de fray Alonso Briceño en su ministerio, consiguieron con pública utilidad de los indios que acudiesen en número de seis mil a la iglesia del convento de Cajamarca" (Urdaneta, 1973, p. 63).

Su inquietud por los indios se refleja claramente cuando desde La Plata escribe el 18 de febrero de 1629 a fray Bernardino de Cárdenas, futuro Obispo de Asunción, indicando su deseo de ir a predicar a los indios:

(...) deseando acudir cuanto es de mi parte al remedio de tan gran dańo y al instituto de nuestro padre san Francisco, que no es vivir para sí solo, sino para provecho de las almas, y también por la obligación que nos corre de acudir en esta(s) materias al descargo de la conciencia de su majestad (Muñoz, 2004, p. 118).

De regreso a Lima para seguir ejerciendo su cátedra en 1636, nuevamente es elegido consejero provincial. Por su gran preparación académica (filosófica y teológica) y religiosa, se le solicita el mismo año que vaya a Roma para presentar la causa de canonización de Francisco Solano -franciscano español, muerto en el Perú-, esgrimiendo los argumentos necesarios para alcanzar prontamente el objetivo: la santificación del fraile menor.

4. El lector franciscano era el profesor que podía enseñar especialmente la doctrina. Los frailes lectores que estaban con los indios -como es el caso en varias ocasiones de fray Alonso Briceño- debían aprender las lenguas vernáculas para poder enseñar mejor a esos desposeídos de la cultura española. A su vez, dos veces jubilado, equivalía al máximo rango académico de la época. En términos de hoy, profesor emérito.

5. "Autor, padre F. Alonso Briceño, Chileno, profesor de Sagrada Teología dos veces jubilado...".

6. Según el Art. 175, $\mathrm{N}^{\circ} 3$ de las Constituciones Generales de la Orden de los Hermanos Menores, guardián es el superior de una casa y la rige "con autoridad ordinaria él solo o con el capítulo local y, respectivamente, en los casos determinados por el derecho, con el Directorio, si lo hay, a tenor de estas constituciones y de los estatutos" (Constitución franciscana).

7. Según el Art. 213 de las Constituciones generales de la orden de los hermanos menores, "el visitador general, elegido por el Ministro general con su definitorio (consejo), visita canónicamente las provincias u otras entidades y, conforme a la regla, amonesta, conforta y corrige caritativamente a los hermanos en nombre y con la autoridad del Ministro General. En la visita procurará conocer las condiciones en que se hallan los hermanos, examinar iniciativas, impulsar actividades y, sobre todo, promover el espíritu de fraternidad y la observancia de nuestra regla y de las constituciones generales" (Constitución franciscana).

8. Equivalente a la segunda autoridad de la provincia religiosa; reemplaza al provincial. 
Llega a Europa llevando sus escritos acerca de la filosofía escotista y logra que en España le publiquen en 1638, en la imprenta real, el primer volumen de su obra Celebriores controversias in primum Sententiarum Ioannis Duns Scoti, Doctoris subtilis Theologorum facile Principis..., ${ }^{9}$ la cual consta de 738 páginas. El segundo volumen, de 565 páginas, aparece en 1642 y el tercero queda inédito. Con estas publicaciones su nombre es reconocido y valorado en la madre patria, especialmente por los entendidos en filosofía, teología, escolástica y metafísica. Estando en España, el Ministro General lo nombra juez de apelaciones de las causas grandes para las provincias de españa, otro importante reconocimiento a su inteligencia y preparación (Urdaneta, 1973).

Lograda la publicación de su primer volumen, viaja a Roma a cumplir la misión encomendada. Allí se entrevista con el papa Urbano VIII y participa en el capítulo general de la orden celebrado en Roma en 1639. Se destaca por la defensa de sus Conclusiones o tesis filosófico-teológicas “(...) en que campeó con tan singular magisterio e inteligencia en la doctrina del Sutil Doctor, ${ }^{10}$ que llenó el crédito que había de su persona" (Urdaneta, 1973, p. 68). Permanece tres años en Roma en donde se le nombra calificador del santo oficio. ${ }^{11}$

De regreso en España, la publicación del segundo volumen hace que no sea considerado de segundo orden por ser americano. Su prestigio llega a oídos del rey Felipe IV, quien lo nombra como su representante en el Capítulo general de los cistercienses o benedictinos. esta institución se había establecido en la Península Ibérica en el siglo XII y para este capítulo asisten las provincias de Madrid, Roma, La Coruña y Valladolid. El encuentro se lleva a cabo en San Benito El Real de Valladolid (Urdaneta, 1973).

Por todo lo anterior y con el beneplácito del Rey de España, quien lo presentó el 5 de junio de 1644, en el consistorio $^{12}$ celebrado en Roma el 14 de noviembre de 1644, el papa Inocencio X, dicta la bula de esa fecha para nombrarlo obispo de Nicaragua - la diócesis abarcaba Nicaragua y Costa Rica-. Es consagrado en la ciudad de Panamá el 12 de noviembre de 1645 por el obispo del lugar, fray Fernando Ramírez, y toma posesión de su obispado en 1646. Allí tiene algunas discusiones por la ciudad en la cual se instalará (León o Granada), ubicándose finalmente en el barrio indígena de Subtiava, cerca de León, al lado de la iglesia que ayudará a construir. En este lugar evangeliza a los naturales en su lengua (mangue) de origen azteca.

Debido a los conflictos suscitados por el lugar de la sede episcopal, el rey, gracias al patronato, ${ }^{13}$ dispuso el traslado del obispo Briceño al obispado de Caracas en 1651. Sin embargo, por la muerte prematura de su predecesor y a otros conflictos, solo el 15 de noviembre de 1653, Inocencio X dicta la bula para asumir la nueva diócesis. Sin embargo, llega a Venezuela en 1660, se instala en Trujillo y allí muere de fiebre el 15 de noviembre de 1668 sin haber tomado posesión de su nueva diócesis de Caracas (Urdaneta, 1973).

En el palacio arzobispal de Caracas, según Urdaneta (1973, p. 95), existe un cuadro del obispo Briceño que describe así su vida:

Religioso menor observante, lector jubilado, teólogo de primer orden y que supo andar todo el estadio de las prelaturas, definiciones, presidencias y visitas de la orden; publicó tratados escolásticos de gran mérito y pasando después a Roma se hizo más visible presidiendo allí Tesis teológicas de rompimiento y apertura de todo un capítulo general celebrado en presencia de los mayores purpurados. Salió de ella calificador y recaló a Nueva España hecho obispo de Nicaragua y de allí a corto espacio trasegó estos mares a obispo de esta Santa Iglesia Catedral de Caracas, año 1660, donde dio los mismos ejemplos de doctrina y piedad que en Lima, Chile, Madrid, Roma y Nueva España. salió a la santa visita, falleció en ella y fue sepultado en la Nueva Trujillo, dejando en ésta, su Iglesia catedral, el testimonio de su vida pastoral hasta su muerte, el $16^{14}$ de noviembre de 1668.

\section{Sus principales obras y su pensamiento filosófico}

Para entender y comprender la trascendencia de la obra filosófica y teológica de fray Alonso Briceño, hay que tener presente que en la Europa de la Edad Media, la filosofía se cultivaba en las universidades, las cuales estaban en manos de instituciones de la Iglesia católica. Algo similar ocurre en la América Colonial. Por lo mismo, frailes, curas o eclesiásticos se dedican a escribir grandes tratados, promoviendo la enseñanza oficial de la filosofía

9. Las célebres controversias en las primeras Sentencias de Juan Duns Escoto, Doctor Sutil, Príncipe de los Teólogos.

10. Apelativo con el cual se le conoce al beato Juan Duns Escoto.

11. El Santo Oficio o la Inquisición era el tribunal eclesiástico que nombraba a un teólogo para censurar libros y proposiciones.

12. Se denomina consistorio a la reunión del Colegio Cardenalicio convocada por el papa para ayudarle en el gobierno de la Iglesia. Los cardenales son creados en los consistorios; en este caso, se firmó la bula para nombrar un obispo en América.

13. El patronato era una facultad concedida por el papa a los reyes que respetaban y promovían el catolicismo en Europa. En este caso, los reyes de Espańa poseían tal facultad y podían solicitar al papa el nombramiento de los obispos o su traslado.

14. Los datos existentes en las otras fuentes señalan al 15 de noviembre, como el día de su muerte. Seguramente fue durante la noche del 15 al 16.

$84<$ Universidad de San Buenaventura, Cali, Colombia 
y practicándola ya sea en cátedras universitarias o en las aulas de los conventos. Quizás por ello, a la filosofía se le menciona como ancilla Theologiae —esclava de la teología-. Efectivamente, es difícil delimitar los campos de ambos saberes. Sin embargo, "una clara excepción sería la obra de Alonso Briceño, quien incluyó en ella, claramente especificados como tales, los temas filosóficos que consideró vinculados con sus tesis teológicas (Muñoz, 2004, pp. 115-116).

Más aún, el pensamiento filosófico de Briceño es considerado no sólo protoexistencialista sino que su filosofía es propiamente americanista (Cacciatore, 2006). Al respecto, Sarti señala:

Si es cierto que las premisas de la especulación briceñiana radican en Europa, también es cierto que las consecuencias que él saca-al sacar a flote el sentido vital, terreno de la existencia, el subrayar la comunión casi entrańable entre el hombre y su mundo, su circunstancia- corresponden profundamente a la mentalidad latinoamericana, como sucesivamente se irá más nítidamente configurando y aclarando (Sarti, 1976, p. 11).

Un gran estudioso de la filosofía hispanoamericana indica:

Briceño saltó desde América al cielo de la metafísica y de la teología. A la diestra de Escoto... Y podía llegar a hacerlo, y otros tras él, porque la doctrina filosófica y teológica de Escoto... es de una tal amplitud y riqueza de sugerencias que su maestro y fundador, lejos de aplastar la originalidad y el surgimiento de pensadores émulos dignos de él, los estimula y favorece (García Bacca, 1954, p. 24).

La gran obra del P. Briceño es la ya mencionada $C e$ lebriores controversias in primum Sententiarum Scoti. El estudio del pensamiento del beato Juan Duns Escoto es algo normal entre los franciscanos. Más aún, en los documentos oficiales de los siglos XVI y XVII se propone en los contenidos generales de enseñanza en los centros franciscanos que se realicen via Scoti ${ }^{15}$ (Manzano, 1992, p. 338). Sin embargo, la profundidad de los escritos de Briceño sobre Escoto, debido a su gran inteligencia y perseverancia, hacen sobresalir a este filósofo chileno sobre todos los demás, transformándose en uno de los más preclaros franciscanos nacidos en América. Por algo se le llama el segundo Escoto. Sus cualidades humanas, sus conocimientos filosófico-teológicos, su exquisita prudencia y su gran celo por la evangelización de los indios, lo hacen destacar entre sus contemporáneos (Merino, 1993). En el primer volumen de su obra insertó una apología de la vida y doctrina del Doctor Sutil — como se le conoce a Escoto-, y un sumario de la santidad y doctrina del beato Amadeo y de otros discípulos o seguidores de Escoto. En el segundo volumen realiza sus comentarios a las célebres controversias filosófico-teológicas del beato inglés. ${ }^{16} \mathrm{Se}$ gún el filósofo español, nacionalizado venezolano, Juan García Bacca,

(...) sus disertaciones, sobre los puntos más importantes de la metafísica de todos los tiempos abarcan: esencia y existencia; unidad del ser: principio de individuación; teoría del conocimiento; potencia natural de la materia; sustancia espiritual; unidad y pluralidad; género; intuición; verdad; objeto del entendimiento; bondad; tiempo; duración; posibilidad, y otras materias de su dilatada sabiduría filosófico-teológica (García Bacca en Urdaneta, 1973, p. 106).

A su vez, en el Art. I de la segunda disputa, al refutar la afirmación tomista de que la unidad del ser consiste en algo positivo, señala:

Porque según Escoto, es muy probable que la hipóstasis ${ }^{17}$ no implique de suyo más que una negación de dependencia actual y aptitudinal; luego, según él, no podrá menos de ser muy probable el que la unidad consista también en la mera negación de división, ya que no aparece razón alguna especial por la que haya que atribuir un concepto positivo a la unidad cuando no conviene ni a la hipóstasis (García Bacca en Caiceo \& Sánchez, 1988, p. 14).

Por su parte, dos destacados tratadistas mexicanos, Francisco Larroyo y Edmundo Escobar, indican un aporte muy importante de la filosofía de Briceño en torno a la haecceidad. ${ }^{18}$ Señalan:

Dentro del itinerario renovador de Escoto, comunica el americano a la doctrina de la haecceidad un sentido con cierto sabor existencialista. El principio de la individuación no es la materia signada por la cantidad, como dijo Santo Tomás, sino la unidad individual, singular, la que tiene cada ser por sí mismo, aquí, ahora. Más da un paso adelante al fundar a la manera de san Agustín tal principio en la experiencia interna del hombre; y puesto en marcha este principio metafísico, llamado así, el de la experiencia interna, recorre todos los clásicos problemas metafísicos. Termina, apoyado en un mitigado voluntarismo, por separar la filosofía de la

15. A través de Escoto.

16. El autor de este texto ha tenido a la vista el único ejemplar en latín que se encuentra en el archivo franciscano de Santiago.

17. Concepto filosófico-teológico, equivalente a persona, central para la comprensión cristiana del misterio de Dios Trinidad, de Jesucristo, Hijo de Dios encarnado, y de la verdad del hombre. Tomás de Aquino, por ejemplo, afirma que la hipóstasis/persona es una substancia intelectual individual, pero añade que es un ser «subsistente (subsistens)" (cf. 5. Th. 1, q. 29, a. 3) «incomunicable" (cf. Ib. ad 4um), o bien una incommunicabilis subsistentia (In Sent.30, 4) (Caiceo, 1992).

18. La haecceidad es un principio de individuación en un plano unívoco de inmanencia. 
Teología, de radical manera (Arroyo y Escobar en Urdaneta, 1973, pp. 113,114).

En el segundo volumen se refiere en una de sus Controversias a la ciencia de Dios. Skarica (1993), filósofo chileno, comenta al respecto que tras considerar la pertinencia de la ciencia en Dios y el objeto formal de la voluntad divina, Briceño hace un estudio especial sobre la ciencia de Dios en relación con los actos libres del hombre, esto es con relación a los futuros contingentes: si Dios puede conocerlos; si los conoce en sí mismos o en sus causas; si la libertad humana queda necesitada por ese conocimiento; cuál es la causalidad y necesidad de la presciencia condicional (Muñoz, 2004).

Avanzando en la originalidad del pensamiento briceñiano, García Bacca observa:

Si tenemos alguna posibilidad de explicar lo que ser yo, y esa su original manera de identificarse con todo, e identificar consigo todo, sin quedar preso y agotado por nada, sólo se puede seguir el camino escotista: identidad fáctica, que entre en la esencia, pero que no llegue a ser de la esencia. Una especie de nuevo parricidio parmenídeo. ${ }^{19}$ Esta vez no contra su concepto de ser, sino contra su principio de identidad. Y ahora tal vez cause demasiada extrańeza que a partir de Escoto y por su influjo -frecuentemente mediante Suárez, ${ }^{20}$ así en Descartes- haya sido posible acometer el tema de la conciencia, del yo: del individuo que se está siendo como singular, y aún como mónada ${ }^{21}$ (García Bacca, 1954: 65/66).

Existe un tercer volumen De Voluntate et Potentia Dei, de Preadestinatione et Trinitate complectens caeteras controversias ad Primum Sententiarum atinentes, que no se publicó, aunque ya estaba escrito.

Respecto a la obra de fray Alonso Briceño existen diversos comentarios. Entre ellos se destacan:

Fue el primero, al decir de González Dávila, que, en América, publicase algo del género de literatura filosófica (José Toribio Medina $^{22}$ en Urdaneta, 1973, p. 130).

Como personaje muy conocido, es una de las figuras más notables de la filosofía y la teología en la época hispánica americana (Ismael Quiles ${ }^{23}$ en Urdaneta, 1973, p. 130).

Compuso todo un tratado de metafísica que puede servir de comentario constante a la filosofía escotista. En el siglo XVII no hay aquí en toda América, ni tal vez, tampoco en Europa, quien le aventaje (Juan David García Bacca ${ }^{24}$ en Urdaneta, 1973, p. 130).

\section{Eduardo Rosales. Vida y pensamiento}

\section{Principales rasgos biográficos}

Nace en San Clemente, comuna de la actual región del Maule, el 25 de junio de 1906, según Iturriaga ${ }^{25}$ (2007) y el 14 de junio, según Olivares ${ }^{26}$ (2003). Sus padres fueron Máximo Rosales y Eloisa Rojas. Desde pequeño desea ingresar a una orden que le permita el estudio. De esta forma, en el convento de Talca tiene el primer encuentro con los franciscanos y comienza sus estudios más profundos con ellos. Realiza su profesión solemne en 1929 y es ordenado sacerdote en 1932. En el seminario de La

19. Se refiere al filósofo griego Parménides

20. Importante filósofo escolástico jesuita.

21. Etimológicamente, derivado del griego, significa "unidad”, "uno", "solo", "único"; la fuente o el Uno. En la antigüedad fue un término para Dios o el primer ser o la unidad originaria, o para la totalidad de todos los seres, con el significado de «sin división». Para Lebnitz en la época moderna, las mónadas son al ámbito metafísico, lo que los átomos, al ámbito físico/fenomenal; las mónadas son los elementos últimos del universo (Leibniz, 1714). Hoy se sostiene que los elementos más pequeños son los quark y leptón.

22. José Toribio Medina Zavala (1852-1930) es bibliógrafo e historiador chileno, considerado el mayor recolector de fuentes para el estudio de la historia del país.

23. Ismael Quiles Sánchez (Valencia, España, 1906-Buenos Aires, 1993) fue un destacado filósofo y sacerdote de la Compañía de Jesús, español que desarrolló su actividad en Argentina; fundador de la Filosofía Insistencial; Decano, Vice-Rector y Pro-Rector de la Universidad del Salvador de Buenos Aires. Estuvo en Chile invitado por el Departamento de Filosofía de la Educación de la Pontificia Universidad Católica de Chile y lo conoció personalmente el autor de este artículo.

24. Juan David García Bacca (Pamplona, España, 1901- Quito, 1992) es un destacado filósofo del Círculo de Viena, lógico, ensayista y traductor español nacionalizado venezolano; pero murió en Ecuador, junto a su esposa de esa nacionalidad, Fanny Palacios; allí descansan sus restos. Este filósofo tradujo los dos tomos de la obra de Briceño.

25. Fray Rigoberto Iturriaga, nacido en Rancagua entre 1970 y 1972, es maestro de novicios en la vicaría de San Miguel de Argentina, Ministro de Provincia e integrante del Consejo de Roma y Asís. Entre 1972 y 1978 es Ministro Provincial de la Provincia de la Santísima Trinidad, Chile. En 1980 es Visitador general de la provincia de Asunción de la Santísima Virgen del Río de la Plata, Argentina. Entre 1982 y 1984 es custodio del Sagrado Corazón, visitador en Argentina y Paraguay. Entre 1985 y 1987 es Vicario provincial, guardián, maestro y párroco de Ossa en Santiago. Visitador en Paraguay (1988) y Perú (1990). Integrante del equipo investigador de la orden en Italia (1991-1993). Ministro Provincial de la Santísima Trinidad, Chile (1993-1999). Vicario de la Recoleta y visitador provincial (2000-2005) (Valenzuela, 2007).

26. Fray Luis Olivares Molina fue doctor en teología egresado de la pontificia universidad católica de chile y doctor en historia de la Iglesia de la Pontificia Pniversidad Gregoriana de Roma. Fue director del instituto de ciencias religiosas durante los ańos 70, miembro del consejo de presbiterio de la diócesis de Valparaíso, y miembro del consejo de énfasis espiritual de la Asociación Cristiana de Jóvenes de Valparaíso (Pontificia Universidad Católica de Valparaíso, 2014).

27. Sacerdote salesiano que ejerce como director espiritual de Raúl Silva Henríquez. Le ayuda en su decisión de ingresar a la Congregación Salesiana; posteriormente, mons. Silva Henríquez se transformará en el segundo cardenal de Chile el 19 de marzo de 1962, creado por el papa san Juan XXIII.

$86<$ Universidad de San Buenaventura, Cali, Colombia 
Granja se inicia su especialización en la moral, la cual la prosigue en sus estudios de doctorado en la Universidad Católica de Chile entre 1936 y 1939. Allí sus profesores son José Blanch, Víctor Panzarraza27 y monseñor Juan Luis Baeza. En 1940 se especializa en psicología en la Universidad Católica de Washington y en algunos centros de Canadá (Olivares, 2003).

Un discípulo y amigo franciscano lo recuerda como un hombre humilde y verdadero maestro. "Siempre estarán frescas en mi mente todas sus enseñanzas, sus normas de vida, su método científico, sus criterios para juzgar hechos, personas y cosas; sus virtudes y sus defectos" (Olivares, 2003, p. 23). Más adelante agrega:

En este momento lo veo junto a mi escritorio, a mis libros, a mis apuntes; con su hábito siempre adornado con algunas manchas, el cordón no del todo blanco, restos de una corona o rosario franciscano; sandalias y cabeza tonsurada... Y siempre sonriendo y mirando al cielo y conversando de los temas más variados... con opiniones muy propias. Ese es el recuerdo que tengo de mi maestro... Siempre discutimos acerca de nuestros diferentes puntos de vista... (Olivares, 2003, p. 24).

En 1939 fray Eduardo Rosales Rojas obtiene el primer grado de doctor que ha otorgado la Universidad Católica de Chile. En este caso en teología y su tema de tesis fue sobre Doctrina acerca de la limosna según san Buenaventura. De eso ya han pasado 80 años y al año 2009 han recibido ese grado académico 762 personas. Cabe señalar que antes de 1969 sólo accedieron a él catorce alumnos y el aumento explosivo se produjo a partir de este siglo (AA., 1970). La Facultad de Teología se funda en la Universidad Católica de Chile solo en 1935 (Caiceo, 1982), por lo tanto, es notable que fray Eduardo Rosales sea el primer alumno en doctorarse. Su preocupación en cultivar su espíritu y perfeccionarse era muy grande. Fue profesor de ética en la misma universidad y, al mismo tiempo, prefecto de estudios en la orden. En el seminario de La Granja dicta Teología Moral y Derecho Canónico. En la Universidad Católica de Chile aparece consignado como profesor de Moral Médica para alumnos de medicina en 1944 (Celis et al., 1982: 99) y Filosofía Moral para alumnas de Educación Familiar entre 1944 y 1952 (Celis et al., 1982, p. 101) y es mencionado entre los filósofos destacados en eventos relacionados con la filosofía, especialmente por su participación en el Congreso de Filosofía en Lima en 1952 (Celis et al., 1982: 104). Según Olivares (2003) también dicta clases de Teología Moral en la Facultad de Teología de la misma universidad. Desgraciadamente debe abandonar la Universidad porque se le acusa que enseña ¡moral sin principios!, a pesar de que su obra más importante en dos tomos publicada en 1961 Hacia una moral social y profesional, señala en el prólogo: "El presente trabajo es fruto de veinte años de profesorado en la Universidad Católica de Chile... He querido dar especial atención a las inquietudes de la juventud. Puedo afirmar que ha sido ella la inspiradora de las líneas generales de este trabajo" (Rosales, 1961, Vol. I, p. 11).

Es extraño, además, tal decisión, pues sus principales obras sobre moral fueron publicadas por la Editorial Universitaria, dependiente de la Universidad de Chile, la cual posee un riguroso comité académico de selección y revisión de sus ediciones.

Al respecto el padre Olivares recuerda que cuando él también tuvo que abandonar las clases de Cultura Religiosa en la Universidad Católica de Valparaíso ¡por enseñar una teología con principios!, fray Rosales le indica:

El franciscano entra a la Universidad por obediencia, nunca porque él lo ha buscado. Y por obediencia debe también dejarla. Y ańadió sonriendo: Por lo demás, sigues la tradición que inició San Buenaventura, y que en Chile continuó el P. León Valenzuela, quien también se vio obligado a dejar sus clases en la Facultad de Teología de Universidad Católica de Chile, jpor enseñar una moral con principios que hoy todo el mundo admite como yo mismo! Y después, silencio absoluto y jamás ningún otro comentario (Olivares, 2003, p. 33).

Participa en otros congresos, como el mariológico de Roma en 1950 y en el de Filosofía Escotista en Oxford y en el de Teología en Roma, ambos en 1966. También está en Roma para la proclamación del dogma de la Asunción de María en cuerpo y alma al cielo por el papa Pío XII, el $1^{\circ}$ de noviembre de 1950 y asiste a algunas sesiones del Concilio Vaticano II y especialmente a su solemne clausura el 8 de diciembre de 1965. Mientras está en Roma muere (21 de noviembre de 1965) su compañero y amigo, el obispo Franciscano de los Ángeles, monseñor Luis Yáńez Ruiz Tagle. Todos estos viajes los hace junto a su discípulo, fray Luis de Tolosa Olivares Molina ${ }^{28}$ (Olivares, 2003).

En su vida como intelectual supo armonizar aquello con su labor pastoral: le gusta confesar en la Iglesia de San Francisco de la Alameda en Santiago, trabajar en la parroquia de La Granja, las misiones populares y la asesoría a movimientos universitarios católicos (Olivares, 2003).

Fallece el 6 de abril de 1970 en la capilla de Ossa, ubicada en Chiloé 1420, Santiago Centro (Iturriaga, 2007). 
Para fray Luis Olivares, su discípulo, con su muerte se cierra una época del intelectual franciscano en Chile o, en otros términos, del testimonio y la presencia de la espiritualidad franciscana en el amplio campo de la ciencia, pues

... el padre Eduardo es el último franciscano que yo conozco que hizo del saber un estilo de vida, y de la ciencia, un apostolado... Murió sońando con el alborear de una nueva edad, en la cual la teología, la filosofía, la historia, es decir, la ciencia, se fusionara con la pastoral en hermosa síntesis. En esa síntesis tan querida a todo verdadero franciscano que de la teología y de la vida hacen una sola cosa, un solo camino hacia Dios; el ideal bonaventuriano expresado en el Itinerarium mentis in Deum ${ }^{29}$ y en el De reductione artium ad theologiam 30 (Iturriaga, 2003, p. 25).

\section{Sus principales obras y su pensamiento filosófico}

Según fray Roberto Iturriaga (2000), Rosales tiene varias publicaciones, destacándose las principales. ${ }^{31}$

Como se puede apreciar en sus escritos, su preocupación investigativa se centra en 1 . asuntos ligados a importantes filósofos de su orden, como San Buenaventura, principal filósofo franciscano de la Escuela de París, y San Antonio de Padua, primer profesor de filosofía de la primera escuela franciscana que se ubica en Bolonia; 2. temas religiosos (congresos y santuarios marianos y franciscanos, especialmente en Italia e Israel), ${ }^{32}$ y 3 . estudios de profundización acerca de la Teodicea y de la filosofía moral. Sobre este último tema se centran especialmente sus actividades docentes, tanto en la orden como en la Universidad Católica de Chile.
Los cambios que se producen a partir de la década de 1960 en la sociedad y las consecuencias del Concilio Vaticano II para la Iglesia, no le agradan al padre Rosales. Sin embargo, guarda silencio e insiste en la postura tradicional respecto a la moral:

La mayoría, si no la totalidad de las preocupaciones que bullen hoy en el campo de la moral no son solamente de hoy; tienen raíces amplias en el pasado inmediato y lejano y, como consecuencia, no tienen para el conocedor de la historia el sentido inquietante que tal vez angustia al que no conoce la historia de la moral (Rosales, 1968, p. 9).

La moral, para el padre Rosales, es la ciencia que busca y señala el camino que el hombre debe seguir para imitar a Cristo. Piensa que después del Concilio Vaticano II debe renovarse, pero en forma fecunda. aAl respecto, señala:

(...) un leal y tesonero esfuerzo por comprender mejor la moral cristiana; por darse (los moralistas) un más claro concepto del misterio del hombre, y así poder presentar la moral de modo que sea comprensible y atrayente, y así logre convertirse en luz y guía del hombre en la difícil tarea de armonizar consigo mismo, con los demás y con los misterios del más allá (Rosales, 1968, p. 69).

El Tomo I de la obra de Rosales (1961: 5), lo dedica a la patria en su sesquicentenario, en los siguientes términos:

La moral anhela hacer hombres perfectos. Su meta es la eternidad. Su campo de acción, el tiempo. El mejor ciudadano, será aquél que vive los imperativos de la moral. Como el presente trabajo va encaminado a facilitar el vivir esos imperativos y por lo tanto a intensificar la excelencia de los ciudadanos, esperanza y grandeza de la patria, a ella se lo ofrecemos con devoción filial.

29. Itinerario del alma -en el sentido de mente- hacia Dios.

30. Reducción de las ciencias a la teología.

31. Doctrina acerca de la limosna según san Buenaventura. Memoria de prueba para optar al título de doctor en Teología de la Pontificia Universidad Católica de Chile. San Francisco de Mostazal: Imprenta Escoto, 1939. Newman y la moral en Anales de la Facultad de Teología, N 6. Santiago de Chile: Universidad Católica de Chile, 1945*. San Antonio de Padua: Ensayos sobre su doctrina. Buenos Aires: Ed. Difusión, 1947. Breve curso de moral. Buenos Aires: Ed. Difusión, 1948. La realeza de María en las Sagradas Escrituras. En: Actas del Congreso Asuncionista Franciscano de América Latina. Buenos Aires, Itinerarium, 1950, pp. 201-230. Universidades católicas y algunos santuarios. Apuntes de viaje referentes a Universidades Católicas y a profesores de Teología Moral, con la reseńa de algunos santuarios de Italia y Palestina. Santiago de Chile: Ed. Universitaria, 1952. San Francisco en algunos de sus santuarios, 1952. Separata del anterior. Hacia una moral social y profesional. Tomos I y II. Santiago de Chile: Prensas de la Editorial Universitaria, 1961. Preocupaciones y actitudes en el campo moral. Santiago de Chile: Editorial Universitaria, 1968. El ateísmo y los fulgores de Dios. Santiago de Chile: Ed. Jurídica Andrés Bello, 1970 **. (s.f.). San Francisco en algunos de sus Santuarios. Santiago de Chile: Editorial Universitaria, s.f. $* * *$

[* Artículo no consignado por Iturriaga, pero señalado por Celis et al., 1982: 197. ** En Iturriaga (2000) se fija como fecha de publicación 1970; en cambio en la Biblioteca Central de la Pontificia Universidad Católica de Chile (1959) se consigna 1969 como año de publicación. ${ }^{* * *}$ Consignado solamente en la Biblioteca Central de la Pontificia Universidad Católica de Chile (1959)].

32. En Caiceo (2018) se señala la intención de San Francisco de Asís de visitar los santos lugares. no pudo concretarlo, pero gracias al Sultán Mélek-elKamel logra que sus hermanos menores ingresen a la antigua Palestina y formen una provincia franciscana conocida como Custodia Franciscana de Tierra Santa. De entre todos los territorios en los cuales los franciscanos tienen actividad, la Custodia de Tierra Santa es, sin duda, uno de los más significativos desde el punto de vista histórico y simbólico. Sus funciones son la animación de la liturgia en los templos de los santos lugares, especialmente Nazaret, Belén, Jerusalén, el Monte Tabor y el Monte Nebo, hecho constatado personalmente por el autor de este artículo, a través de la coordinación de las iglesias locales, la recepción de los peregrinos provenientes de todas partes del mundo para orar en ellos, la asistencia en el sostenimiento de las estructuras que allí se encuentran, como así también actividades ecuménicas, docentes y de investigación bíblica-arqueológica; las instituciones académicas de referencia son el Studium Biblicum Franciscanum de Jerusalén y el Instituto Arqueológico Franciscano (Monte Nebo, Jordania) (Caiceo, 2014). De ahí la importancia que fray Eduardo Rosales les da a estos lugares y a Italia, especialmente a Asís, pues allí se encuentra el origen de la orden. 
Su preocupación por las nuevas ideologías desde inicios de la segunda mitad del siglo pasado, lo lleva a la teodicea. En el fondo se encuentra preocupado por las nuevas inquietudes del pensamiento humano. Dice al respecto:

Se concibió este trabajo, gracias a una circunstancia personal: las inquietudes ideológicas de los últimos años. Sobre todo la insistencia con que se habla del ateísmo, cual si fuera una fuerza irresistible que todo lo avasalla, me causó temores. Dejé de lado los estudios de moral y me di de nuevo a repensar lo que había estudiado desde que sentí la inclinación a la vida religiosa. Con especial interés traté de aquilatar todo lo que se refiere a la existencia de Dios, porque todo el edificio cristiano descansa ahí (Rosales, 1970, p. 287).

\section{Conclusiones}

Al llegar al final de este trabajo se puede constatar que se ha dado cumplimiento a su objetivo. En efecto, se ha entregado una visión general de la vida de los dos filósofos franciscanos chilenos y se ha profundizado en su obra filosófica, especialmente en el caso de fray Alfonso Briceño, considerado el segundo Escoto en el pensamiento filosófico franciscano. Su nivel de profundidad es asombroso, especialmente en el ámbito metafísico y como indica Sarti (1976), en lenguaje latinoamericano. Por lo mismo, es reconocido como uno de los más importantes filósofos de Latinoamérica por autores como Cacciatore, García Bacca, Manzano, Merino, Muñoz, Quiles, Skarica y Urdaneta. En el caso de fray Eduardo Rosales, él se distinguió por su docencia a nivel universitario y en la formación de miembros de su orden y por centrarse en sus escritos en la moral social y profesional.

A modo de conclusión, se indica que los filósofos franciscanos poseen un "horizonte mental que los distingue de otras escuelas" (Merino, 1993, p. XXVII). Lo fundamental radica en que "una cierta experiencia'personal y comunitaria está en la base del franciscanismo. Aquí la teoría y el sistema son el resultado de una vivencia y de una praxis condicionantes del pensamiento" (Merino, 1993, p. XXVII). Efectivamente, la praxis latinoamericana está presente en el planteamiento metafísico de fray Alfonso Briceño y el abordaje de la moral de fray Eduardo Rosales.

\section{Referencias}

AA. (2010). Constitución Franciscana en http://www.franciscanos.org/docoficial/ constituciongohm7-8.htm. Consultado el 16 de mayo de 2014.
AA. (1970). "Doctores en la Universidad Católica de Chile" en http://www.latercera.com/contenido/675_203741_9. shtml. Consultado el 29 de junio de 2014.

Biblioteca Central de la Pontificia Universidad Católica de Chile (1959). Bibliografia eclesiástica chilena. Santiago de Chile: Editorial Universidad Católica.

Cacciatore, Giuseppe (2006). "La escolástica española y la génesis de la filosofía latinoamericana. alonso Briceño: Metafísica e Individualidad". En: Limite. Revista de Filosofía y Psicología, Vol. I, No 14, pp. 5-24.

Caiceo, Jaime (1982). "Presencia de la filosofía en los anales de la Facultad de Teología”. En: Anales Facultad de Teología, Vol. XXXIII. Santiago de Chile, Pontificia Universidad Católica de Chile, pp. 179-188.

Caiceo, Jaime (1992. En torno al problema del ser. Santiago de Chile: Ediciones Instituto Profesional de Estudios Superiores Blas Cañas.

Caiceo, Jaime (2016). "Presencia franciscana en las universidades durante los siglos XIII y XIV”. En: Ágora, V. 16, N ${ }^{\circ}$ 2, pp. 603-622.

Caiceo, Jaime (2018). Filosofia franciscana: principales autores en sus textos. $2^{\mathrm{a}}$ edición, Santiago de Chile: Colegio Santa Isabel de Hungría, Imprenta Innovación Gráfica.

Caiceo, Jaime; Sánchez, Elena (1988). Clarence Finlayson. Sinopsis de la filosofia en Chile. Santiago de Chile: Facultad de Filosofía de la Pontificia Universidad Católica de Chile.

Celis, Luis; Caiceo, Jaime; López, Sara; Sánchez, Elena; Aliaga, Fernando; Court, Ignacio. (1982). "La presencia de la filosofía en la Universidad Católica: 18881973”. En: Anales Escuela de Educación, No. 5. Santiago de Chile: Pontificia Universidad Católica de Chile, pp. 1-215.

Directorio Franciscano (s.f). San Francisco de Asis en http:// www.franciscanos.org/sfa/ menud.html. Consultado el 4 de octubre de 2019.

Escárate, Alexis, ofm. (2014) "Presentación y reflexión en torno al libro Filosofia franciscana: principales autores en sus textos de Jaime Caiceo Escudero" en Anuario, 2014. Santiago de Chile: Colegio Santa Isabel de Hungría, pp. 140-146.

García Bacca, Juan (1954). Antología del pensamiento filosófico venezolano. Caracas: Ministerio de Educación.

Iturriaga, Rigoberto (2000) Impresos franciscanos. Santiago de Chile: Publicaciones del Archivo Franciscano No 66.

Iturriaga, Rigoberto (2007) 4268 Nombres y otros datos... Elenco de religiosos de la orden franciscana que han pasado por Chile. Santiago de Chile: Publicaciones del Archivo Franciscano, $\mathrm{N}^{\circ} 100$.

Leibniz, Gottfried (1714). Discours de Métaphysique: Monadologie. París: Tel Galdstard. 
Manzano, Isidoro, ofm (1992). “Alonso Briceño (1587-1668): Franciscano, Pensador, Obispo". En: Archivum Franciscanum Historicum, Grottaferrata (Roma), An. 85, pp. 333-366.

Merino, José Antonio (1993). Historia de la filosofia franciscana. Madrid: Biblioteca de Autores Cristianos.

Muñoz García, Ángel (2004). "Alonso Briceño, filósofo de Venezuela y América”. En: Patio de Letras, Año II, vol. II, No 1, pp. 115-130.

Olivares, Luis, ofm (2003). "El primer doctor de la Facultad de Teología de la Pontificia Universidad Católica de Chile: fray Eduardo Rosales Rojas, ofm”. En: Anuario de historia de la Iglesia en Chile, Volumen 21. Santiago de Chile: Seminario Pontificio Mayor, pp. 23-39.

Pontificia Universidad Católica de Valparaíso (2014). Noticias. 20 de agosto en http://prensa.ucv.cl/?p=35180. Consultado el 4 de octubre de 2019.
Rosales, Eduardo ofm (1961). Hacia una moral social y profesional, Tomo I. Santiago de Chile: Prensas de la Editorial Universitaria.

Rosales, Eduardo ofm (1968). Preocupaciones y actitudes en el campo moral. Santiago de Chile: Editorial Universitaria.

Rosales, Eduardo ofm (1970). El ateísmo y los fulgores de Dios. Santiago de Chile: Ed. Jurídica Andrés Bello.

Skarica, Mario (1993) "Predeterminación y libertad en fray Alonso Briceño”. En: Philosophica, N 16, Universidad Católica de Valparaíso, pp. 57-63.

Urdaneta, Ramón (1973). Alonso Briceño. Primer filósofo de América. Caracas: Universidad Católica Andrés Bello.

Valenzuela, Alexis (2007). "fray Roberto Iturriaga Carrasco recibe Medalla Cruz de Triana” en http://www.elrancahuaso.cl/admin/render/ noticia/12486 del 31 de diciembre. Consultado el 4 de octubre de 2019. 